\title{
REDUCTION OF STRIPING NOISE IN OVERLAPPING LIDAR INTENSITY DATA BY RADIOMETRIC NORMALIZATION
}

\author{
Wai Yeung Yan, Ahmed Shaker \\ Department of Civil Engineering, Ryerson University, Toronto, Ontario, Canada \\ (waiyeung.yan ahmed.shaker)@ryerson.ca
}

Commission VI, WG VII/4

KEY WORDS: LiDAR Intensity, Noise Reduction, Radiometric Correction, Radiometric Normalization, Land Cover Classification

\begin{abstract}
:
To serve seamless mapping, airborne LiDAR data are usually collected with multiple parallel strips with one or two cross strip(s). Nevertheless, the overlapping regions of LiDAR data strips are usually found with unbalanced intensity values, resulting in the appearance of stripping noise. Despite that physical intensity correction methods are recently proposed, some of the system and environmental parameters are assumed as constant or not disclosed, leading to such an intensity discrepancy. This paper presents a new normalization technique to adjust the radiometric misalignment found in the overlapping LiDAR data strips. The normalization technique is built upon a second-order polynomial function fitted on the joint histogram plot, which is generated with a set of pairwise closest data points identified within the overlapping region. The method was tested on Teledyne Optech's Gemini dataset (at $1064 \mathrm{~nm}$ wavelength), where the LiDAR intensity data were first radiometrically corrected based on the radar (range) equation. Five land cover features were selected to evaluate the coefficient of variation $(c v)$ of the intensity values before and after implementing the proposed method. Reduction of $c v$ was found by $19 \%$ to $59 \%$ in the Gemini dataset, where the striping noise was significantly reduced in the radiometrically corrected and normalized intensity data. The Gemini dataset was also used to conduct land cover classification, and the overall accuracy yielded a notable improvement of $9 \%$ to $18 \%$. As a result, LiDAR intensity data should be pre-processed with radiometric correction and normalization prior to any data manipulation.
\end{abstract}

\section{INTRODUCTION}

The use of airborne LiDAR data has progressively increased for surface classification and object recognition (Yan et al., 2015). Despite that, there still exist several knowledge gaps limiting the use of LiDAR intensity data. Among which the striping noise appeared in the overlapping region of mosaicked LiDAR intensity data causes undesired disturbance, and such visual detrimental effect undoubtedly degrades the radiometric quality of the data. Regardless of discrete-return or full-waveform LiDAR data, such intensity noise is mainly caused by the signal attenuation due to various system and environmental factors (Jelalian 1992). Though various correction and calibration techniques have been proposed to reduce the intensity discrepancy based on the use of radar (range) equation (Höfle and Pfeifer, 2007, Kaasalainen et al. 2009, Wagner, 2010, Yan et al. 2012), only a few studies address the striping noise issue when dealing with the overlapping LiDAR data strips.

Luzum et al. (2004) proposed a method to normalize the observed LiDAR intensity by multiplying a dynamic range factor to the power of $f(f=2)$, where such dynamic range factor equals to the range of the observed point divided by a standard range. Such dynamic range normalization method has been enhanced and used to normalize multiple overlapping LiDAR data strips, particularly for forest canopies, with a notable improvement in terms of classification accuracy (Korpela et al. 2010a b, Gatziolis, 2011). Despite that, the method has certain drawbacks which limit its applicability in a universal environment. The selection of $f$ (or the two calibration parameters: $a$ and $b$ in (Korpela et al. 2010a b) ) highly depends on the nature of the study site (target characteristics) and the LiDAR sensors (Hopkinson 2007, Korpela et al. $2010 \mathrm{a}$ b). In addition, the method does not consider other system and environment parameters except the range effect; therefore the method is preferable to be implemented with LiDAR dataset col- lected for rugged forest terrain within small scan angle (less than $10^{\circ}$ to $15^{\circ}$ ). The lack of consideration of incidence angle would lead to intensity discrepancy, which can be found particularly serious in urban environment with inclined rooftops (Jutzi and Gross 2010, Abed et al. 2012, Yan and Shaker 2014). Though there exists preliminary attempts to incorporate Phong model in the radar (range) equation for overlap data strip correction (Ding et al. 2013), Jutzi and Gross (2010) addressed that the Phong model does not really outperform the traditional Lambertian assumption in terms of intensity homogeneity.

Joint histogram (also comparagram or scattergram) is a two dimensional matrix, which describes the occurrence of any pairwise intensity value found within two images. Joint histogram has been used in image processing and computer vision, including image comparison (Pass and Zabih. 1999), change detection (Kita. 2006), image registration (Lu et al. 2008), and estimation of camera response function (Kim and Pollefeys, 2008). With any two geo-registered images taken for the same scene, a robust mapping function can be defined in the joint histogram plot so that the relationship between the intensity values of the two images can be established with a fitted regression model (Mann. 2000). As inspired by these studies, we propose an alternative normalization technique for overlapping LiDAR intensity data based on the use of joint histogram technique. Unlike digital image data, it is nearly impossible to identify any laser footprints of two overlapping LiDAR data strips projected at the same location. Therefore, we first identify all possible pairwise LiDAR data points within a threshold distance, and then generate the intensity joint histogram. A polynomial curve is subsequently fitted and used as a transformation function to normalize the intensity from a LiDAR data strip to a reference LiDAR data strip. Our ultimate goal is to develop a fast and robust method to normalize any two overlapping LiDAR data strips and significantly reduce the striping noise appeared in the mosaicked intensity image. 


\section{METHOD}

\subsection{Overall Workflow}

Fig. 1] shows the overall workflow of the proposed normalization method. In general, the proposed method can be applied to any entirely or partially overlapping LiDAR intensity data. Firstly, if those system and environmental parameters (i.e. range, scan angle, atmospheric attenuation coefficients, etc.) are available, radiometric correction can be applied to the original intensity (OI) data (Yan et al. 2012, Yan and Shaker 2014). Conceptually, the spectral reflectance (or radiometrically corrected intensity (RCI)) is determined for each of the LiDAR data strips $\left(\mathbf{X}^{\mathbf{A}}\right.$ and $\left.\mathbf{X}^{\mathbf{B}}\right)$ after radiometric correction. Such RCI derived from both LiDAR data strips can be used to generate a joint histogram by searching for all possible pairwise closest points, and a polynomial function is subsequently fitted in the joint histogram. The fitted polynomial curve is being treated as an intensity transformation function to normalize the data strip $\mathbf{X}^{\mathbf{B}}$ with reference to the $\mathbf{X}^{\mathbf{A}}$. After radiometric normalization, the (partially or fully) overlapping intensity data strips are interpolated to generate an intensity image. To measure the degree of intensity noise, we compute the coefficient of variation of selected land cover features and compare the intensity homogeneity between the OI and the radiometrically corrected and normalized intensity data.

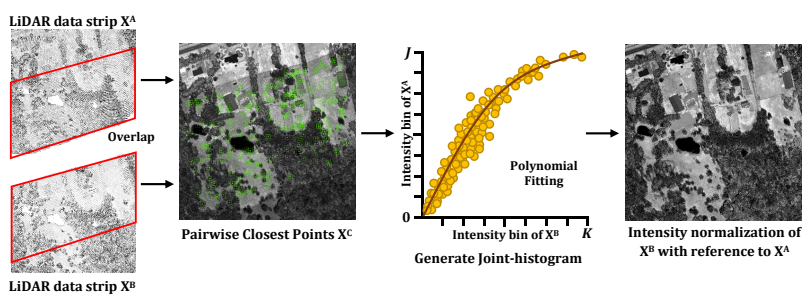

Figure 1: Overall Workflow

\subsection{Radiometric Correction}

Various radiometric correction and calibration techniques have been developed for discrete-return or full-waveform LiDAR data based on the radar (range) equation (Höfle and Pfeifer. 2007. Kaasalainen et al. 2009, Wagner. 2010, Jutzi and Gross, 2010. Yan et al. 2012). The purpose of radiometric correction aims to retrieve the surface reflectance of the illuminated object for each of the received laser pulses. As shown in Eq. 1. the radar (range) equation describes the relationship between the received laser power $\left(P_{r}\right)$ with respect to various system and environmental parameters:

$$
P_{r}=\frac{P_{t} D_{r}^{2}}{4 \pi R^{4} \beta_{t}} \eta_{s y s} \eta_{a t m} \sigma
$$

where $P_{t}$ is the transmitted laser pulse energy, $D_{r}$ is the aperture diameter, $R$ is the range, $\beta_{t}$ is the laser beam width, $\eta_{\text {sys }}$ is the system factor, and $\eta_{a t m}$ is the atmospheric attenuation factor. The laser cross section $\sigma$ consists of the illuminated surface characteristics that can be expressed as $\sigma=4 \pi \rho A \cos \theta$, where $A$ is the projected target area along the direction of the laser beam, $\theta$ is the laser incidence angle, and $\rho$ is the spectral reflectance of the illuminated surface. In Eq. 1 the surface reflectance $\rho$ is being treated as the radiometrically corrected intensity data, and the original intensity $I$ is assumed to be directly proportional to the transmitted laser pulse $P_{t}$. In order to retrieve the surface reflectance $\rho$, the aforementioned parameters, if known, can be inputted in the Eq. 1. and those parameters which are unknown can be assumed as constant. Since effect of overcorrection has been reported when laser incidence angle is used in radiometric correction, a combined use of scan angle and incidence angle can be adopted to resolve such an issue (Yan and Shaker 2014).

\subsection{Radiometric Normalization}

After radiometric correction, the intensity data of two partially overlapping LiDAR datasets are used to generate the joint histogram so as to perform a robust normalization. Unlike georeferenced image, it is mostly impossible to find a pairwise LiDAR data point that are situated at the same position in a three dimensional Euclidean space. Therefore, our proposed algorithm has to first locate the closest LiDAR data points from two overlapping LiDAR data strips within a threshold distance. The proposed method first utilizes a LiDAR data strip with a larger intensity range as a reference, i.e. $\mathbf{X}^{\mathbf{A}}=\left\{x_{1}^{A}, \cdots, x_{a}^{A}, \cdots, x_{N_{A}}^{A}\right\}$, and then any LiDAR data strip, i.e. $\mathbf{X}^{\mathbf{B}}=\left\{x_{1}^{B}, \cdots, x_{b}^{B}, \cdots, x_{N_{B}}^{B}\right\}$, with partial/entire overlap can be normalized with reference to $\mathbf{X}^{\mathbf{A}}$. For each $x_{a}^{A} \in \mathbf{X}^{\mathbf{A}}$, we look for the closest point in $\mathbf{X}^{\mathbf{B}}$ whose Euclidean distance $d=\left\|x_{a}^{A}-x_{b}^{B}\right\|_{2}$ is smaller than a given threshold $d_{\min } . y_{a}^{B}$ thus denotes the resulting closest point where:

$$
y_{a}^{B}=\arg \min \left\{d\left(x_{a}^{A}, x_{b}^{B}\right)\right\}, \text { subject to } d<d_{\text {min }}
$$

The resulting $N_{C}$ correspondence pairwise LiDAR data points from $\mathbf{X}^{\mathbf{A}}$ and $\mathbf{X}^{\mathbf{B}}$ are denoted as:

$$
\mathbf{X}^{\mathbf{C}}=\left\{\left(x_{a}^{A}, y_{a}^{B}\right) \mid i=1, \cdots, N_{C}\right\}
$$

Then, the intensity values of $x_{a}^{A}$ and $y_{a}^{B}$ are used to generate the joint histogram $\mathbf{H} . J$ and $K$ are the total number of intensity bins of data strip $\mathbf{X}^{\mathbf{A}}$ and $\mathbf{X}^{\mathbf{B}}$, respectively, provided that $J \geq K . N(j, k)$ is the number of corresponding pairs of LiDAR data points in $\mathbf{X}^{\mathbf{C}}$ having intensity value $j$ in $x_{a}^{A}$ and $k$ in $y_{a}^{B}$. Therefore, the joint histogram $\mathbf{H}$ is a form of a $J$ by $K$ matrix:

$$
\mathbf{H}=\left[\begin{array}{ccc}
N(0,0) & \cdots & N(0, K-1) \\
N(1,0) & \vdots & N(1, K-1) \\
\vdots & N(j, k) & \vdots \\
N(J-2,0) & \vdots & N(J-2, K-1) \\
N(J-1,0) & \cdots & N(J-1, K-1)
\end{array}\right]
$$

where

$$
N_{C}=\sum_{j=0}^{J-1} \sum_{k=0}^{K-1} N(j, k)
$$

The intensity transformation function should be in a form of linear relationship or piecewise linear relationship (Yan and Shaker. 2014). Thus, a polynomial model can be used as an approximate solution to transform the intensity value of $\mathbf{X}^{\mathrm{B}}$ to $\mathbf{X}^{\mathrm{A}}$.

\section{EXPERIMENTAL WORK}

\subsection{Study Area and Dataset}

The study area is located in the east of the Newmarket, Greater Toronto Area, Ontario, Canada. The extent is bounded by the Davis Drive to the north, Highway 48 to the east, Vivian Road to the south, and Kennedy Road to the west, resulting in a dimension of $4.7 \mathrm{~km}$ by $3.5 \mathrm{~km}$. Within the immediate vicinity of the study area, farmlands, grasslands, mixed forested area (deciduous and coniferous), small houses and paved roads exist. The 
proposed approach was examined on two LiDAR datasets collected by Teledyne Optech's sensors. The first dataset, including two data strips, was collected by the Teledyne Optech's Gemini operating at $1064 \mathrm{~nm}$ wavelength. The flight mission was accomplished on August $24^{\text {th }}, 2013$, where the air temperature and atmospheric pressure were $20^{\circ} \mathrm{C}$ and 1027.4 millibars, respectively. The Gemini sensor was operated with scan frequency 40 $\mathrm{Hz}$, scan angle $\pm 20^{\circ}$, pulse repetition frequency $70 \mathrm{kHz}$ and flying attitude $1,000 \mathrm{~m}$. With these settings, the mean point density yields 3.7 points $/ \mathrm{m}^{2}$ for the two data strips collected. Table 1 summarizes the LiDAR system settings and data specification.

Table 1: LiDAR system settings and data specification

\begin{tabular}{|c|c|}
\hline & Dataset \\
\hline \hline Sensor & Gemini \\
\hline Date of Acquisition & ${\text { August } 24^{\text {th }}, 2013}^{2}$ \\
\hline Number of Data Strips & $1064 \mathrm{~nm}$ \\
\hline Wavelength & $\sim 1,000 \mathrm{~m}$ \\
\hline Flying Height & $40 \mathrm{~Hz}$ \\
\hline Scan Frequency & $\pm 20^{\circ}$ \\
\hline Scan Angle & $70 \mathrm{kHz}$ \\
\hline Pulse Repetition Frequency & $\sim 3.7$ points $/ \mathrm{m}^{2}$ \\
\hline Mean Point Density & $\sim 1 \mathrm{~m}$ \\
\hline Mean Point Spacing & $\sim 41 \%$ \\
\hline Percentage of Overlap & \\
\hline
\end{tabular}

\subsection{Implementation}

Since the LiDAR data provided were stored in las format, the las files were first converted into ASCII data format using LAStools. The following fields were read from the las files: $x, y, z, I, a$, $r, n$, and time, where they represent $\mathrm{x}$-coordinate, $\mathrm{y}$-coordinate, z-coordinate, intensity, angle, return, number of returns, and GPS time, respectively. The converted ASCII text files were then imported into ArcGIS geodatabase as 3D point features. On the other hand, two individual GPS trajectory files (in 8-byte data format) were read for the two LiDAR datasets in order to retrieve the $x y z$ coordinates and GPS time of the aircraft during the flight missions. By interpolating the GPS time of the aircraft and the LiDAR data points, instantaneous aircraft coordinates were computed for each of the data points in the two LiDAR datasets. The range and incidence angle of each LiDAR data point were computed by following the method presented in Yan and Shaker (2014). Then, radiometric correction was applied to all the LiDAR data strips for both datasets.

In the process of radiometric normalization, a set of pairwise closest points should be first identified in the overlapping region of the two data strips. We used the "Near 3D" tool in ArcGIS to look for the closest point $y_{a}^{B}$ from $\mathbf{X}^{\mathrm{B}}$ to pair with a $x_{a}^{A}$ from $\mathbf{X}^{\mathbf{A}}$ (refer to section 2.3 for detail). As a result of computation, the 3D distance for each pairwise match was computed in $\mathbf{X}^{\mathrm{A}}$ together with the unique identity of the closest point in $\mathrm{X}^{\mathrm{B}}$. Finally, we only selected those pairwise points from $\mathbf{X}^{\mathbf{A}}$ and $\mathbf{X}^{\mathbf{B}}$ with a threshold distance (i.e. $d_{\min }$ in section 2.3 less than 5 $\mathrm{cm}$. A larger threshold value than that would result in a scattered shape in the joint histogram plot, and thus increases the values in the residual matrix $\mathbf{V}$ in Eq. ??. The polynomial fitting was implemented in the joint histogram plot, and the coefficients of the polynomial function were computed using MATLAB. Fig. 2 shows the joint histogram plots fitted with a polynomial curve for each of the Teledyne Optech's datasets. Subsequently, radiometric normalization was applied to the data strip $\mathrm{X}^{\mathrm{B}}$ in ArcGIS, and then both $\mathbf{X}^{\mathbf{A}}$ and normalized $\mathbf{X}^{\mathbf{B}}$ were merged and converted into an intensity image using a $3 \times 3$ moving window average method (Reuter et al. 2007).

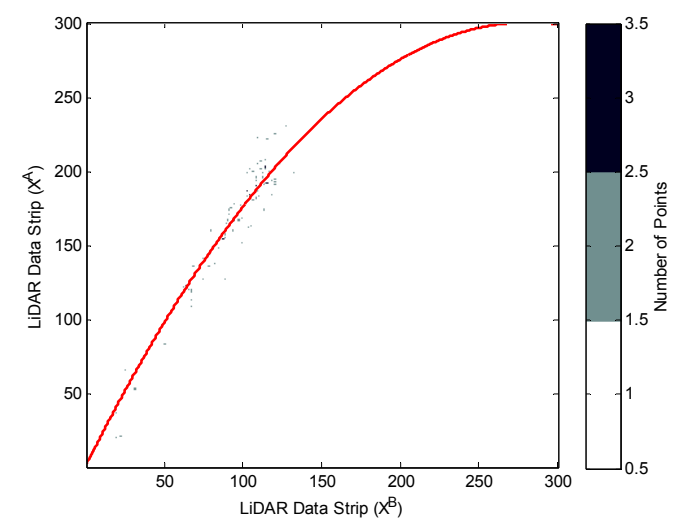

Figure 2: Joint histogram plot fitted with polynomial function

\subsection{Design of Experiments}

Two rounds of experiments were conducted on the Gemini datasets in order to test the capability of the proposed method. Firstly, we combined the original intensity data of the two overlapping data strips to form an original intensity image, denoted as OI. We then implemented the radiometric correction and normalization on the two data strips and generated an intensity image, named as RCNI. Apart from visual inspection on the intensity images, a statistical measure, coefficient of variation $(c v)$, was used to assess the intensity homogeneity of selected land cover feature $\left(\omega_{i}\right)$.

$$
c v\left(\omega_{i}\right)=\frac{\sigma\left(\omega_{i}\right)}{\mu\left(\omega_{i}\right)}
$$

In this context, a smaller $c v$ corresponds to a less within-class variation of intensity in the land cover sample points. If radiometric correction and normalization can significantly reduce the striping noise within the intensity data, a reduction of $c v$ value should be recorded.

\section{RESULTS AND ANALYSIS}

Fig. 3 shows the study area located at the south of the intersection of Highway 48 and Vivian Road. As shown in Figs. 3(a) and $3(\mathrm{e})$ both OI images had serious striping noise in the cross track direction within the overlapping region. The intensity values located in the bottom half of the image were lower than that of the upper half of the image, resulting in an unbalanced image contrast within the OI. The proposed radiometric correction and normalization successfully removed the striping noise and spikes, and restored the intensity image close to a balanced contrast (see Figs. 3(b) and 3(f)p. Despite that, it can be noticed that a low level of striping noise still retained on some of the rooftops in the RCNI.

To further analyze the results, we utilized the $c v$ as a quantitative measure of image quality improvement (Vovk et al. 2007). We computed the $c v$ of five selected land cover features for all the datasets, where a reduction of $c v$ indicates an improvement of intensity homogeneity, resulting in a less noisy intensity image. Table 2 shows the $c v$ computed for the OI and RCNI for five selected land cover features. The most significant reduction of $c v$ was achieved in ground features, i.e. bare ground, grass and road. The $c v$ of $\mathrm{OI}$ in bare ground was 0.473 ; a reduction of $59 \%$ was found in the RCNI data. Similar reduction of $c v$ was recorded in grass and road features, where a respective decrease of $65 \%$ and $48 \%$ was found in the RCNI data. Although the percentage of $c v$ reduction in those elevated features was not comparable to the 


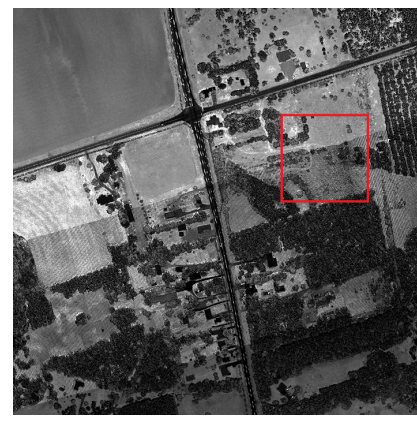

(a) $\mathrm{OI}$

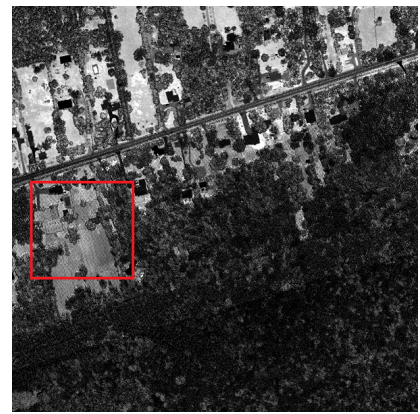

(e) $\mathrm{OI}$

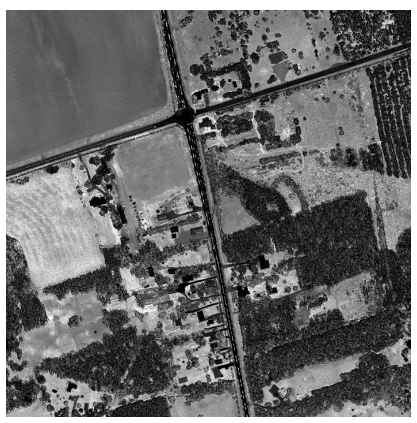

(b) RCNI

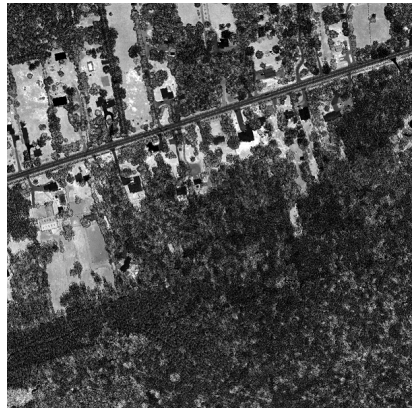

(f) $\mathrm{RCNI}$

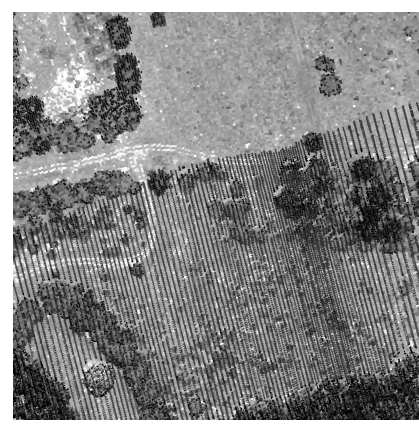

(c) Squared area in OI

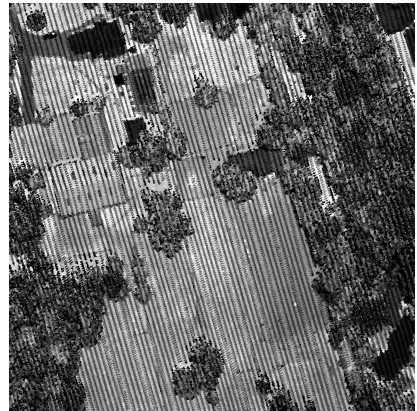

(g) Squared area in OI

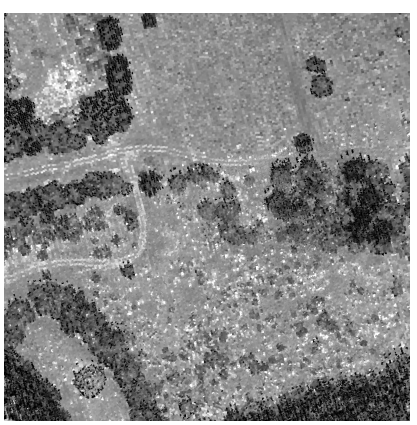

(d) Squared area in RCNI

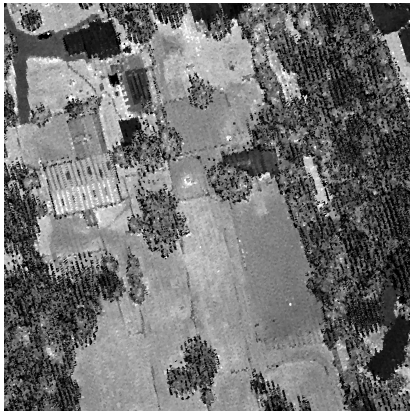

(h) Squared area in RCNI

Figure 3: LiDAR intensity image of Gemini (1064 nm)

previous two classes, the RCNI still recorded a $19 \%$ reduction of $c v$ in the tree canopies, and a $25 \%$ of decrease on the house rooftops. With both visual examination and statistical analysis, the proposed radiometric correction and normalization can significantly reduce the striping noise appeared in the overlapping region.

Table 2: A comparison of $c v$ of selected land cover features.

\begin{tabular}{|c|c|c|}
\hline & OI & RCNI \\
\hline \hline Bare ground & 0.473 & $0.193(\downarrow 59 \%)$ \\
\hline Grass & 0.475 & $0.168(\downarrow 65 \%)$ \\
\hline House & 0.591 & $0.443(\downarrow 25 \%)$ \\
\hline Road & 0.584 & $0.304(\downarrow 48 \%)$ \\
\hline Tree & 0.839 & $0.676(\downarrow 19 \%)$ \\
\hline
\end{tabular}

\section{LAND COVER CLASSIFICATION}

In order to demonstrate the impact of the striping noise reduction, we used the OI shown in Fig. 3(g) and RCNI shown in Fig. 3(h) to perform land cover classification, and compared their results. As depicted in Figs. 3(g) and 3(h), land cover features including trees, grass cover, paved driveway/road, houses and a warehouse can be found in the study site. Therefore, we identified these five land cover classes for training site selection, and implemented the classification with different combinations of features: 1) intensity data only, 2) intensity and digital surface model (DSM), 3 ) intensity and texture (TEX) features generated from the intensity, and 4) intensity, TEX and DSM. Previous studies reported that the use of entropy texture and homogeneity texture can significantly contribute to the enhancement of classification accuracy (Samadzadegan et al. 2010, Huang et al., 2011); therefore, these two texture features were generated for both OI and RCNI with a window size of $9 \times 9$ to support the experimental testing. A total of eight classification scenarios were implemented by using the traditional maximum likelihood classifier, and 1,000 random checkpoints were generated to assess the classification results. Table 3 summarizes the overall accuracy generated for all the eight classification scenarios.

Table 3: Overall accuracy of LiDAR data classification results.

\begin{tabular}{|c|c|c|}
\hline & OI & RCNI \\
\hline \hline Intensity Only & $24.3 \%$ & $42.4 \%(\uparrow 18.1 \%)$ \\
\hline Intensity+DSM & $56.0 \%$ & $65.0 \%(\uparrow 9.0 \%)$ \\
\hline Intensity+TEX & $52.8 \%$ & $69.9 \%(\uparrow 17.1 \%)$ \\
\hline Intensity+TEX+DSM & $69.3 \%$ & $83.5 \%(\uparrow 14.2 \%)$ \\
\hline
\end{tabular}

Although only using OI or RCNI led to an accuracy lower than $50 \%$, the overall accuracy produced by RCNI outperformed than that of OI with an improvement of $18.1 \%$. In the second classification scenario, the difference of overall accuracy between OI and RCNI was reduced. The OI+DSM produced an accuracy of $56 \%$, while the RCNI+DSM improved the overall accuracy up to $65 \%$ resulting in an $9 \%$ improvement. This can be explained by the use of elevation data which can aid in differentiating the grass cover from the tree canopies as well as the paved driveway from the house rooftop. In case of using the entropy and homogeneity textures, the overall accuracy achieved by the OI+TEX was $52.8 \%$, where the classification result of RCNI+TEX noted an $17.1 \%$ improvement (almost reached to $70 \%$ ). Due to the striping noise found in the OI, the texture features generated would retain high variance in most of the land cover features. This thus leads to a low separability among the land cover classes, which can be proven in the $c v$ values of OI as shown in table 2 The last scenario demonstrated the classification result produced by the intensity, textures and DSM, which yielded the best performance among all the classification scenarios. The combination of RCNI+TEX+DSM produced an overall accuracy of $83.5 \%$, comparing to that of OI+TEX+DSM which was $69.3 \%$ only. Based on our experimental testing, the LiDAR intensity data processed with radiometric correction and normalization achieves a notable improvement in land cover classification, regardless of the feature space being used. Such an accuracy improvement can be ascribed 
by the reduction of striping noise, and therefore the proposed method should be applied to the overlapping LiDAR data strips before performing any surface classification and object recognition.

\section{CONCLUSIONS}

This paper presents a radiometric normalization technique to reduce the striping noise appeared in the overlapping region of airborne LiDAR intensity data strips. The normalization model is built upon the use of a $2^{\text {nd }}$ order polynomial function fitted on a joint histogram plot, which is generated based on a set of pairwise intensity data points identified within the overlapping LiDAR data strips. After applying the proposed method on two datasets (Teledyne Optech's Gemini) with wavelength $1064 \mathrm{~nm}$, the striping noise was significantly reduced in the intensity images. To quantitatively assess the results, we adopted the coefficient of variation as a statistical measure to assess the intensity homogeneity. The experimental results showed that the $c v$ was reduced by $19 \%$ to $65 \%$ in the radiometrically corrected and normalized intensity data. We also tested the capability of using LiDAR intensity data to perform land cover classification with different combinations of feature spaces. The results showed that an accuracy improvement ranging from $9 \%$ to $18 \%$ was achieved in classifying five land cover classes, when the LiDAR intensity data were pre-preprocessed with radiometric correction and normalization. The experiments prove that radiometric correction and normalization not only reduce the striping noise visually and quantitatively, but also lead to a notable improvement of overall accuracy when using the intensity data for land cover classification. The proposed method does not require any selection of parameters or reference targets, and thus overcomes those drawbacks found in the existing normalization techniques based on the dynamic range factor. For large scale seamless mapping, radiometric normalization can be applied to the multiple parallel LiDAR data strips with reference to the cross data strip(s). With slight modification, the proposed method can also be implemented on mobile LiDAR data and multispectral LiDAR data.

\section{ACKNOWLEDGEMENTS}

The research was supported by the Natural Sciences and Engineering Research Council of Canada (NSERC) Engage Grant. The authors thank Teledyne Optech Inc., in particular Dr. Joe Liadsky and Dr. Paul LaRocque, for providing the airborne LiDAR intensity data as well as their constructive comments on the research work.

\section{References}

Abed, F. M., Mills, J. P. and Miller, P. E., 2012. Echo amplitude normalization of full-waveform airborne laser scanning data based on robust incidence angle estimation. IEEE Transactions on Geoscience and Remote Sensing 50(7), pp. 2910-2918.

Ding, Q., Chen, W., King, B., Liu, Y. and Liu, G., 2013. Combination of overlap-driven adjustment and phong model for lidar intensity correction. ISPRS Journal of Photogrammetry and Remote Sensing 75, pp. 40-47.

Gatziolis, D., 2011. Dynamic range-based intensity normalization for airborne, discrete return LiDAR data of forest canopies. Photogrammetric Engineering \& Remote Sensing 77(3), pp. 251-259.
Höfle, B. and Pfeifer, N., 2007. Correction of laser scanning intensity data: data and model-driven approaches. ISPRS Journal of Photogrammetry and Remote Sensing 62(6), pp. 415-433.

Hopkinson, C., 2007. The influence of flying altitude, beam divergence, and pulse repetition frequency on laser pulse return intensity and canopy frequency distribution. Canadian Journal of Remote Sensing 33(4), pp. 312-324.

Huang, X., Zhang, L. and Gong, W., 2011. Information fusion of aerial images and LIDAR data in urban areas: vector-stacking, re-classification and post-processing approaches. International Journal of Remote Sensing 32(1), pp. 69-84.

Jelalian, A. V., 1992. Laser radar systems. Artech House.

Jutzi, B. and Gross, H., 2010. Investigations on surface reflection models for intensity normalization in airborne laser scanning (ALS) data. Photogrammetric Engineering \& Remote Sensing 76(9), pp. 1051-1060.

Kaasalainen, S., Hyyppä, H., Kukko, A., Litkey, P., Ahokas, E., Hyyppä, J., Lehner, H., Jaakkola, A., Suomalainen, J., Akujarvi, A. et al., 2009. Radiometric calibration of LiDAR intensity with commercially available reference targets. IEEE Transactions on Geoscience and Remote Sensing 47(2), pp. 588-598.

Kim, S. J. and Pollefeys, M., 2008. Robust radiometric calibration and vignetting correction. IEEE Transactions on Pattern Analysis and Machine Intelligence 30(4), pp. 562-576.

Kita, Y., 2006. Change detection using joint intensity histogram. In: The 18th International Conference on Pattern Recognition, 2006, Vol. 2, IEEE, pp. 351-356.

Korpela, I., Ørka, H. O., Hyyppä, J., Heikkinen, V. and Tokola, T., 2010a. Range and AGC normalization in airborne discretereturn LiDAR intensity data for forest canopies. ISPRS Journal of Photogrammetry and Remote Sensing 65(4), pp. 369-379.

Korpela, I., Ørka, H. O., Maltamo, M., Tokola, T. and Hyyppä, J., 2010b. Tree species classification using airborne LiDAR - Effects of stand and tree parameters, downsizing of training set, intensity normalization, and sensor type. Silva Fennica 44(2), pp. 319-339.

Lu, X., Zhang, S., Su, H. and Chen, Y., 2008. Mutual information-based multimodal image registration using a novel joint histogram estimation. Computerized Medical Imaging and Graphics 32(3), pp. 202-209.

Luzum, B., Starek, M. and Slatton, K. C., 2004. Normalizing ALSM intensities. Geosensing Engineering and Mapping (GEM) Center Report No. Rep_2004-07-01. Civil and Coastal Engineering Department, University of Florida, 8pp.

Mann, S., 2000. Comparametric equations with practical applications in quantigraphic image processing. IEEE Transactions on Image Processing 9(8), pp. 1389-1406.

Pass, G. and Zabih, R., 1999. Comparing images using joint histograms. Multimedia Systems 7(3), pp. 234-240.

Reuter, H. I., Nelson, A. and Jarvis, A., 2007. An evaluation of void-filling interpolation methods for SRTM data. International Journal of Geographical Information Science 21(9), pp. 983-1008. 
Samadzadegan, F., Bigdeli, B. and Ramzi, P., 2010. A multiple classifier system for classification of LIDAR remote sensing data using multi-class SVM. In: Multiple classifier systems, Springer, pp. 254-263.

Vain, A., Kaasalainen, S., Pyysalo, U., Krooks, A. and Litkey, P., 2009. Use of naturally available reference targets to calibrate airborne laser scanning intensity data. Sensors 9(4), pp. 27802796.

Vovk, U., Pernuš, F. and Likar, B., 2007. A review of methods for correction of intensity inhomogeneity in MRI. IEEE Transactions on Medical Imaging 26(3), pp. 405-421.

Wagner, W., 2010. Radiometric calibration of small-footprint full-waveform airborne laser scanner measurements: basic physical concepts. ISPRS Journal of Photogrammetry and Remote Sensing 65(6), pp. 505-513.

Yan, W. Y. and Shaker, A., 2014. Radiometric correction and normalization of airborne LiDAR intensity data for improving land cover classification. IEEE Transactions on Geoscience and Remote Sensing 52(10), pp. 7658-7673.

Yan, W. Y., Shaker, A. and El-Ashmawy, N., 2015. Urban land cover classification using airborne LiDAR data: A review. Remote Sensing of Environment 158, pp. 295-310.

Yan, W. Y., Shaker, A., Habib, A. and Kersting, A. P., 2012. Improving classification accuracy of airborne LiDAR intensity data by geometric calibration and radiometric correction. ISPRS Journal of Photogrammetry and Remote Sensing 67, pp. 35-44. 